\title{
Inferential erotetic logic meets inquisitive semantics
}

\author{
Andrzej Wiśniewski • Dorota Leszczyńska-Jasion
}

Received: 16 December 2012 / Accepted: 30 September 2013 / Published online: 8 April 2015

C The Author(s) 2015. This article is published with open access at Springerlink.com

\begin{abstract}
Inferential erotetic logic (IEL) and inquisitive semantics (INQ) give accounts of questions and model various aspects of questioning. In this paper we concentrate upon connections between inquisitiveness, being the core concept of INQ, and question raising, characterized in IEL by means of the concepts of question evocation and erotetic implication. We consider the basic system InqB of INQ, remain at the propositional level and show, inter alia, that: (1) a disjunction of all the direct answers to an evoked question is always inquisitive; (2) a formula is inquisitive if, and only if it evokes a yes-no question whose affirmative answer expresses a possibility for the formula; (3) inquisitive formulas evoke questions whose direct answers express all the possibilities for the formulas, and (4) each question erotetically implies a question whose direct answers express the possibilities for the direct answers to the implying question.
\end{abstract}

Keywords Logic of questions - Inferential erotetic logic - Inquisitive semantics

\section{Introduction}

This paper focuses on interrelations between two notions: question raising and inquisitiveness. The former is in the centre of attention of inferential erotetic logic (IEL), while the latter is the core concept of inquisitive semantics (INQ).

Work on this paper was supported by funds of the National Science Centre, Poland (DEC-2012/04/A/HS1/00715).

A. Wiśniewski $(\varangle) \cdot$ D. Leszczyńska-Jasion

Department of Logic and Cognitive Science, Institute of Psychology,

Adam Mickiewicz University in Poznań, Szamarzewskiego 89, 60-568 Poznan, Poland

e-mail: Andrzej.Wisniewski@amu.edu.pl

D. Leszczyńska-Jasion

e-mail: Dorota.Leszczynska@amu.edu.pl 


\subsection{Inquisitive semantics}

Inquisitive semantics originated from an analysis of questions, but currently evolves toward a general theory of meaning. The beginnings of INQ date to the late 1990s. INQ, however, is currently a research programme rather than a completed theory: alternative accounts are still being proposed. ${ }^{1}$

The basic idea of INQ can be briefly expressed as follows: the meaning of a sentence comprises two components, informative content and inquisitive content. The former is the information provided by a sentence, the latter is the issue raised by the sentence. By and large, if the information provided is sufficient to settle the issue that is raised, the sentence is an assertion. If, however, the information provided is insufficient to settle the raised issue, the sentence is inquisitive.

For example, the following:

$$
\text { Mary is Peter's mother. }
$$

raises the issue whether Mary is Peter's mother and provides information that Mary is Peter's mother. The information provided settles the issue raised. Now let us consider:

$$
\text { John is Peter's father or George is Peter's father. }
$$

Sentence (2) raises the issue who is Peter's father, John or George, while the information provided amounts only to the claim that one of above possibilities holds. So (2) is inquisitive, while (1) is not. Observe that inquisitive sentences are akin to questions in being carriers of information to be completed.

Both types of contents, as well as the concepts of possibility, assertion and inquisitiveness are modelled formally (see below).

In what follows we concentrate upon the "basic", most often used system of inquisitive semantics, labelled as InqB, and we remain at the propositional level only.

\subsection{Inferential erotetic logic}

Generally speaking, IEL is a logic that analyses inferences in which questions play the role of conclusions and proposes criteria of validity for these inferences. The idea originates from the late $1980 \mathrm{~s}^{2}$

IEL starts with a simple observation that in many cases arriving at questions resembles coming to conclusions: there are premises involved and some inferential thought processes take place. In other words, there exist erotetic inferences, that is, thought processes in which questions occur as "conclusions". These inferences are of (at least)

\footnotetext{
1 For various versions of INQ see, e.g., Groenendijk and Roelofsen (2009), Ciardelli and Roelofsen (2011), Groenendijk (2011), Ciardelli et al. (2013a,b), Ciardelli and Roelofsen (2014). For recent developments see: http://sites.google.com/site/inquisitivesemantics/.

2 The monograph Wiśniewski (1995) summarizes results obtained until early nineties, while the book Wiśniewski (2013) provides a state-of-the-art exposition of IEL. For a concise introduction see Wiśniewski (2001) or Wiśniewski (1996).
} 
two kinds, depending on the type of premises involved. In the case of an erotetic inference of the first kind the set of premises consists of declarative sentence(s) only, and an agent passes from it to a question. For example:

(I) Mary is Peter's mother.

If Mary is Peter's mother, then John is

Peter's father or George is Peter's father.

Who is Peter's father: John or George?

The premises of an erotetic inference of the second kind comprise a question and possibly some declarative sentence(s). For instance:

(II) Where did Andrew leave for?

If Andrew took his famous umbrella, then he left for London; otherwise he left for Paris or Moscow.

Did Andrew take his famous umbrella?

Erotetic inferences in which no declarative premise occurs can be regarded as a special case of erotetic inferences of the second kind. Here is an example of an appropriate erotetic inference which does not rely on any declarative premise:

(III) Did Andrew travel by BA, or by Ryanair, or by neither?

Did Andrew travel by BA?

Some erotetic inferences are intuitively valid, while others are not. The following can serve as a preliminary test of intuitive validity: put the expression "so the question arises" just before the conclusion. If the resultant description of an erotetic inference is undoubtedly true, the inference can be regarded as intuitively valid. The reader is advised to perform the test with respect to the examples of erotetic inferences presented above, and then with respect to:

(IV) Mary is Peter's mother.

If Mary is Peter's mother, then John is Peter's father.

Is John Peter's father?

and

(V) Where did Andrew leave for?

If Andrew took his famous umbrella, then he left for London;

otherwise he left for Paris or Moscow.

Is Andrew smart enough?

Clearly, (I), (II) and (III) pass the test, in contradistinction to (IV) or (V). However, there are cases in which one does not get indisputable results. On the other hand, validity is a normative notion, and, as long as erotetic inferences are concerned, is given neither by God nor by Tradition. So some arbitrary decisions have to be made. 
IEL proceeds as follows. First, some criteria of validity are proposed, separately for each kind of erotetic inference (for details see, e.g., Wiśniewski 2013, Chapter 5). Then two semantic relations are defined: evocation of questions by sets of declarative sentences/formulas, and erotetic implication of a question by a question together with a set of declarative sentences/formulas. Validity of erotetic inferences of the first kind is defined in terms of evocation; at the same time the definition of evocation provides an explication of the intuitive notion "a question $Q$ arises from a set of declarative sentences $X$ ". As for erotetic inferences of the second kind, their validity is defined in terms of erotetic implication (of the question being the conclusion by the question which is the premise on the basis of the declarative premise(s) involved). The proposed definition of erotetic implication is an explication of the intuitive notion "a question $Q$ arises from a question $Q_{1}$ on the basis of a set of declarative sentences $X$ ". ${ }^{3}$ In view of IEL question raising (explicated in terms of evocation and erotetic implication) and validity of erotetic inferences are two sides of the same coin.

Both erotetic implication and question evocation are purely semantic concepts. In order to define them one needs, inter alia, a certain concept of entailment for declaratives. However, in its general setting IEL is neutral in the controversy concerning what "The Logic" of declaratives is. One can use Classical Logic, but non-classical logics are also permitted. In this paper we will be dealing with the simplest case, that is, with evocation and erotetic implication based on Classical Propositional Logic.

\subsection{IEL versus InqB}

IEL and InqB are conceptually incommensurable. IEL distinguishes questions from declaratives syntactically, while InqB does not do this. InqB defines the basic semantic categories in terms of support, being a relation between a formula and a set of possible worlds/an information state. IEL, in turn, operates with the concept of truth as the basic one. However, despite conceptual differences, both theories give accounts of the phenomenon of question/issue raising, though the phenomenon is viewed by them from different angles. InqB explicates the intuitive notion of a possibility "offered" by a sentence, distinguishes sentences which offer at least two possibilities and, in effect, gives a semantic characteristics of expressions that call for supplementary information. However, InqB does not provide a systematic account of arriving at issues/questions. IEL, in turn, focuses on semantic relations between questions and the contexts of their appearance. So it seems that IEL and INQ can benefit from their meeting, both conceptually and in further developments. In this paper we present some formal results which may be viewed as arguments in favour of the above claim.

\subsection{Outline of the paper}

The perspective adopted in this paper is the following. As in IEL, and unlike InqB, we consider a (propositional) language which has two disjoint categories of

\footnotetext{
3 Let us stress: IEL does not provide analytic definitions of the above concepts of "arising", but their explications (in the sense of Carnap; see Carnap 1950, pp. 1-18). For details see Wiśniewski (1995, Chapter 1).
} 
well-formed expressions: declarative well-formed formulas and questions. The latter are defined purely syntactically. In other words, the language has a "declarative part" and an "erotetic part". We supplement the declarative part (but not the erotetic part!) with an inquisitive semantics which is, basically, InqB. Since the standard concepts of truth and entailment are definable in InqB, this step enables us to operate with inquisitive as well as standard semantic concepts, in particular to define "classical" question evocation and erotetic implication.

In Sect. 2 we provide an exposition of the basics of InqB and we introduce the semantic concepts used throughout the paper. In Sect. 3 we describe how questions are conceptualized in InqB and in IEL, and we point out some affinities between the so-called proper questions and inquisitive formulas of a special form. Section 4 is devoted to question evocation and its connections with inquisitiveness. We show that a disjunction of all the direct answers to an evoked question is always inquisitive, and that the evoked questions are just the questions whose disjunctions of asserted direct answers are inquisitive. We prove that a formula is inquisitive if, and only if the formula evokes a yes-no question whose affirmative answer expresses a possibility for the formula. We also show that an inquisitive formula evokes a question whose direct answers express all the possibilities for the formula. In Sect. 5 we turn to erotetic implication, explain the underlying intuitions and present the definition. Then we prove that each question (of the language considered) erotetically implies a question whose direct answers express all the possibilities for the direct answers to the implying question.

\section{InqB}

\subsection{Possible worlds, states, and support}

As for InqB, we consider a (non-modal) propositional language $\mathcal{L}_{\mathcal{P}}$ over a non-empty set of propositional variables $\mathcal{P}$, where $\mathcal{P}$ is either finite or countably infinite. The primitive logical constants of the language are: $\perp, \vee, \wedge, \rightarrow$. Well-formed formulas (wffs) of $\mathcal{L}_{\mathcal{P}}$ are defined as usual.

The letters $A, B, C, D$, with or without subscripts, are metalanguage variables for wffs of $\mathcal{L}_{\mathcal{P}}$, and the letters $X, Y$ are metalanguage variables for sets of wffs of the language. The letter $\mathrm{p}$ is used below as a metalanguage variable for propositional variables.

$\mathcal{L}_{\mathcal{P}}$ is supposed to be associated with the set of suitable possible worlds, $\mathcal{W}_{\mathcal{P}}$, being the model of $\mathcal{L}_{\mathcal{P}}$. A possible world, in turn, is conceived either as a subset of $\mathcal{P}$ or as a valuation of $\mathcal{P}$. Regardless of which of these solutions is adopted, $\mathcal{W}_{\mathcal{P}}$ is uniquely determined. When possible worlds are conceptualized as sets of propositional variables, $\mathcal{W}_{\mathcal{P}}=\wp(\mathcal{P})$, that is, $\mathcal{W}_{\mathcal{P}}$ is the power set of $\mathcal{P}$. If, however, possible worlds are identified with indices, that is, valuations of $\mathcal{P}$, then $\mathcal{W}_{\mathcal{P}}$ is the set of all indices.

Subsets of $\mathcal{W}_{\mathcal{P}}$ are called states. States are thus sets of possible worlds. One can think of such sets as modelling information states. Singleton sets/states correspond to information states of maximal consistent information, while $\mathcal{W}_{\mathcal{P}}$ corresponds to the ignorant state, i.e. an information state in which no possible world is excluded. $\varnothing$ represents the absurd state.

The letters $\sigma, \tau, \gamma$, with or without subscripts, will refer to states. 
The most important semantic relation between states and wffs is that of support. In the case of InqB support, $\models$, is defined by:

Definition 1 (Support) Let $\sigma \subseteq \mathcal{W}_{\mathcal{P}}$.

1. $\sigma \vDash \mathrm{p}$ iff for each $w \in \sigma: \mathrm{p}$ is true in $w$,

2. $\sigma \vDash \perp$ iff $\sigma=\emptyset$,

3. $\sigma \vDash(A \wedge B)$ iff $\sigma \vDash A$ and $\sigma \vDash B$,

4. $\sigma \vDash(A \vee B)$ iff $\sigma \vDash A$ or $\sigma \vDash B$,

5. $\sigma \vDash(A \rightarrow B)$ iff for each $\tau \subseteq \sigma$ : if $\tau \vDash A$ then $\tau \vDash B$.

When $\mathcal{W}_{\mathcal{P}}$ comprises indices, " $\mathrm{p}$ is true in $w$ " means "the value of $\mathrm{p}$ under $w$ equals 1". When $\mathcal{W}_{\mathcal{P}}=\wp(\mathcal{P})$, the meaning is: " $p \in w$ ".

Inquisitive negation is introduced by:

$$
\neg A={ }_{d f}(A \rightarrow \perp)
$$

Thus we get:

(neg) $\sigma \vDash \neg A$ iff for each $\tau \subseteq \sigma$ such that $\tau \neq \emptyset: \tau \not \models A$.

The definition of support by a state generalizes the standard definition of truth in a world. To see this, it suffices to put $\sigma=\{w\}$ and then replace " $\{w\} \vDash A$ " with " $A$ is true in $w$ ". We get the usual clauses defining truth of a wff in a world. However, the generalization is non-trivial. Support by a state does not amount to truth in each world of the state: the clauses for disjunction and implication (and also negation) are more demanding. The following holds:

Lemma 1 (Persistence) If $\sigma \vDash A$, then $\tau \vDash A$ for any $\tau \subseteq \sigma$.

As a consequence we get:

Corollary 1 If $\sigma \vDash A$, then $\{w\} \vDash A$ for each $w \in \sigma$.

However, the converse of Corollary 1 does not hold. As an illustration, consider a state $\{w, v\}$ such that $p$ is true in $w, q$ is false in $w, p$ is false in $v$ and $q$ is true in $v$. The wff $p \vee q$ is true both in $w$ and in $v$, but, due to clauses (1) and (4) of Definition 1, $\{w, v\}$ does not support $p \vee q$.

The truth set of a wff $A$, in symbols: $|A|$, is the set of all the worlds from $\mathcal{W}_{\mathcal{P}}$ in which $A$ is true, where the concept of truth is understood classically. More formally, $|A|=\left\{w \in \mathcal{W}_{\mathcal{P}}:\{w\} \vDash A\right\}$.

2.2 Meaning, inquisitiveness, and possibilities

In view of INQ the meaning of a sentence has two aspects: informative content and inquisitive content. Roughly, the former is identified with the information provided, while the latter is the issue raised.

The informative content of a wff $A$, info(A), is defined as follows: 
Definition 2 (Informative content) info( $A)=\bigcup\left\{\sigma \subseteq \mathcal{W}_{\mathcal{P}}: \sigma \vDash A\right\}$

The inquisitive content of a wff $A,[A]$, is defined by:

Definition 3 (Inquisitive content) $[A]=\{\sigma \subseteq$ inf $o(A): \sigma \vDash A\}$

Since support is understood in InqB in the sense of Definition $1,{ }^{4}$ we get:

$$
\inf o(A)=|A|
$$

that is, the informative content of $A$ equals the truth set of $A .^{5}$ It follows that in the case of InqB we have:

$$
[A]=\{\sigma \subseteq|A|: \sigma \vDash A\}
$$

The empty set is regarded as a state and thus, by Corollary 1 and Definition 3, $[A]$ is a non-empty downward closed set of subsets of $|A|$. Inquisitive semantics considers such sets as propositions expressed by wffs. Note that the concept of proposition is "lifted": propositions conceived in this way are not sets of possible worlds, but sets of states, that is, sets of sets of possible worlds.

When one starts with the concept of proposition as the basic one (i.e. the proposition expressed by $A$ construed as a non-empty downward closed set of subsets of $|A|$ ), the structure $\langle\Gamma, \subseteq\rangle$, where $\Gamma$ is the set of all propositions that belong to $\wp\left(\mathcal{W}_{\mathcal{P}}\right)$, is a Heyting algebra with a (pseudo)complementation operation ${ }^{\circ}$ characterized by: $\mathcal{A}^{\circ}={ }_{d f}\{\beta: \beta \cap \bigcup \mathcal{A}=\varnothing\}$, and relative pseudocomplementation, $\Longrightarrow$, defined by: $\mathcal{A} \Longrightarrow \mathcal{B}={ }_{d f}\{\alpha:$ for every $\beta \subseteq \alpha$, if $\beta \in \mathcal{A}$ then $\beta \in \mathcal{B}\}$. This provides an algebraic justification for the clauses of the definition of support. For details see Roelofsen (2013).

A wff $A$ is inquisitive when, and only when its informative content is not an element of its inquisitive content, that is, the (full) informative content of $A$ does not support $A$. To be more precise:

Definition 4 (Inquisitiveness) A wff $A$ is inquisitive iff info $(A) \not \models A$.

For InqB, however, this reduces to:

$$
|A| \notin[A] .
$$

Wffs which are not inquisitive are called assertions.

Here are examples of inquisitive wffs: ${ }^{6}$

\footnotetext{
4 There are systems of inquisitive semantics in which support is defined differently; see Groenendijk (2011), Ciardelli et al. (2013a, b), Ciardelli and Roelofsen (2014).

5 In more traditional terms: the informative content of $A$ amounts to the proposition expressed by $A$.

${ }^{6}$ As for (3), $|p \vee q|=\left\{w \in \mathcal{W}_{\mathcal{P}}: w(p)=\mathbf{1}\right.$ or $\left.w(q)=\mathbf{1}\right\}$. But $|p \vee q| \not p p \vee q$, since it is neither the case that $|p \vee q| \vDash p$ nor it is the case that $|p \vee q| \vDash q$. (Recall that $|p \vee q| \vDash p$ iff $w(p)=\mathbf{1}$ for each $w \in|p \vee q|$, and similarly for $q$. Neither of these cases hold.) Concerning (4): clearly $|p \vee \neg p|=\mathcal{W}_{\mathcal{P}}$. But, again, we neither have $w(p)=\mathbf{1}$ for every $w \in \mathcal{W}_{\mathcal{P}}$ nor we have $w(p) \neq \mathbf{1}$ for each world $w$ belonging to a non-empty subset of $\mathcal{W}_{\mathcal{P}}$. Hence $|p \vee \neg p|$ does not support $p \vee \neg p$ and thus $|p \vee \neg p| \notin[p \vee \neg p]$, that is, (4) is inquisitive.
} 


$$
\begin{aligned}
& p \vee q \\
& p \vee \neg p
\end{aligned}
$$

Observe that (4) is a classical tautology, i.e. $|p \vee \neg p|=\mathcal{W}_{\mathcal{P}}$. However, not every classical tautology is inquisitive-for example $\neg(p \wedge \neg p)$ is not. Thus inquisitive semantics enables a differentiation among tautologies: some of them are inquisitive, while other are assertions.

When we remain at the propositional level, InqB-inquisitive contents of wffs have maximal elements, called possibilities. To be more precise:

Definition 5 (Possibility) A possibility for wff $A$ is a state $\sigma \subseteq \mathcal{W}_{\mathcal{P}}$ such that $\sigma \vDash A$ and for each $w \notin \sigma: \sigma \cup\{w\} \not \forall A$.

If a wff is inquisitive, the set of possibilities for the wff comprises at least two elements.

For example, let us consider:

$$
(p \vee q) \vee \neg(p \vee q)
$$

The set of possibilities for (5) is:

$$
\{|p|,|q|,|\neg p| \cap|\neg q|\}
$$

Note that $|p|$ and $|q|$ (partially) overlap.

It can be proven that each state supporting a wff $A$ is a subset of some possibility for $A{ }^{7}$ Hence it follows that, in particular, each world that belongs to the truth set of a wff $A$ belongs to some possibility for $A$ (see also Lemma 5 below).

\subsection{Entailment}

Entailment is defined in INQ in terms of inclusion: $A$ (inquisitively) entails $B$ iff info( $A) \subseteq \operatorname{info(B)}$ and $[A] \subseteq[B]$. As for InqB, however, $[A] \subseteq[B]$ yields info $(A) \subseteq$ info $(B)$. Thus in the case of InqB inquisitive entailment, $==_{i n q}$, can be defined by:

Definition 6 (Inquisitive entailment) $X=_{i n q} A$ iff $\bigcap_{B \in X}[B] \subseteq[A]$.

Note that the underlying idea is the transmission of support. Needless to say, classical entailment, $\models_{c l}$, can be defined as follows:

Definition 7 (Classical entailment) $X \models_{c l} A$ iff $\bigcap_{B \in X}|B| \subseteq|A|$.

Clearly, the following holds:

Corollary $2 \models{ }_{i n q} \subset \models c l$.

$\overline{7}$ This result may be found in Ciardelli (2009, pp. 9-10), and Ciardelli and Roelofsen (2011, pp. 59-60). 
We also need the concept of multiple-conclusion entailment, ${ }^{8}$ being a relation between sets of wffs. Roughly, $X$ multiple-conclusion entails (mc-entails for short) $Y$ if, and only if the truth of all the wffs in $X$ warrants that at least one wff in $Y$ is true. In the case of Classical Propositional Logic we express the idea by:

Definition 8 (Classical mc-entailment) $X \mid \models_{c l} Y$ iff $\bigcap_{B \in X}|B| \subseteq \bigcup_{A \in Y}|A|$.

\section{Questions}

Generally speaking, questions/interrogatives can be incorporated into a formal language in two ways.

1. (The "DEFINE WITHIN" approach.) One can embed questions into a language. To be more precise, one can regard as questions some already given well-formed formulas differentiated by some semantic feature(s), or construe questions as meanings of some specific (but already given) well-formed formulas.

This way of proceeding is natural when the so-called paraphrase approach to questions is adopted, that is, it is believed that the meaning of an interrogative sentence can be adequately characterized by a paraphrase that specifies the typical use of the sentence or the relevant illocutionary act performed in uttering the sentence. For example, one can claim that the following:

Does John like Mary?

is synonymous with:

Bring it about that I know whether John likes Mary.

or with:

I request that you assert that John likes Mary or deny that he likes Mary.

The paraphrase (8) can be formalized within a setting which involves epistemic operators and imperative operator(s). (9), in turn, can be formalized within a logical theory of illocutionary acts. In both cases no separate, primary syntactic category of interrogatives is needed. For convenience, one can then define "interrogative formulas", but they will be only abbreviations of their counterparts, and, what is more important, their semantics is just the semantics of the relevant well-formed formulas. ${ }^{9}$

\footnotetext{
8 For this concept see, e.g., Shoesmith and Smiley (1978).

9 (8) is a Hintikka-style paraphrase, while (9) agrees with Vanderveeken's approach to questions. The survey paper Harrah (2002) provides a comprehensive exposition of logical theories of questions elaborated up until late 1990s. Supplementary information about more linguistically oriented approaches can be found, e.g., in Groenendijk and Stokhof (1997) (reprinted as Groenendijk and Stokhof 2011) and Krifka (2011). The paper Ginzburg (2011) provides a survey of recent developments in the research on questions, both in logic and in linguistics. A general overview of approaches to questions and their semantics can also be found in Wiśniewski (2015).
} 
2. (The "ENRICH WITH" approach.) One can enrich a language with questions/ interrogatives. In order to achieve this, one adds to the vocabulary some questionforming expressions and then introduces questions/interrogatives syntactically, as a new category of well-formed formulas. The new category is disjoint with the remaining categories. This way of proceeding is natural when questions are conceived in accordance with the independent meaning thesis, according to which the meaning/semantic content of an interrogative sentence cannot be adequately characterized in terms of semantics of expressions that belong to other categories.

IEL proceeds in accordance with the "enrich with" approach, while InqB adopts the "define within" approach. ${ }^{10}$ However, InqB postulates no paraphrase of questions in terms of expressions of other categories; questions and assertions are distinguished semantically among expressions which are traditionally construed as declaratives (see below).

\subsection{Questions in InqB}

The language $\mathcal{L}_{\mathcal{P}}$ does not include a separate syntactic category of questions/ interrogatives. However, some wffs are regarded as having the property of being a question, or $\mathcal{Q}$-property for short. Recall that $\mathcal{W}_{\mathcal{P}}$ stands for the model of $\mathcal{L}_{\mathcal{P}}$, and $|A|$ for the truth set of wff $A$ in $\mathcal{W}_{\mathcal{P}}$ (cf. Sect. 2.1).

Definition 9 (Being a question) A wff $A$ of $\mathcal{L}_{\mathcal{P}}$ has the $\mathcal{Q}$-property iff $|A|=\mathcal{W}_{\mathcal{P}}$.

The $\mathcal{Q}$-property is simply the complement of informativeness defined as follows:

Definition 10 (Informativeness) A wff $A$ of $\mathcal{L}_{\mathcal{P}}$ is informative iff $|A| \neq \mathcal{W}_{\mathcal{P}}$.

Hence a wff $A$ is (i.e. has the property of being) a question when, and only when $A$ is not informative, that is, $A$ is true in each possible world of $\mathcal{W}_{\mathcal{P}}$. Thus the wffs having the $\mathcal{Q}$-property are just classical tautologies. Yet, as we have already observed, InqB enables a differentiation among classical tautologies: some of them are inquisitive, and some are not. So there exist inquisitive questions and non-inquisitive questions.

Recall that the set of possibilities for an inquisitive wff has at least two elements. So if an inquisitive wff, $A$, has the $\mathcal{Q}$-property (i.e. "is" a question), then there exist at least two possibilities for $A$ and, by non-informativeness, the truth set of $A$ equals $\mathcal{W}_{\mathcal{P}}$.

One can easily prove that $A$ has the $\mathcal{Q}$-property iff $A$ and $A \vee \neg A$ are inquisitively equivalent, i.e. inquisitively entail each other. The interrogative operator, ?, is introduced by:

$$
? A={ }_{d f} A \vee \neg A
$$

\footnotetext{
10 Yet, there exist systems of INQ which adopt the "enrich with" approach; see Groenendijk (2011), Ciardelli et al. (2013b), Ciardelli and Roelofsen (2014).
} 
One should not confuse (10) with a syntactic characterization of questions/ interrogatives. Also, it is not claimed that each question is a yes-no question. Since the $Q$-property is the complement of informativeness, no question of $\mathcal{L}_{\mathcal{P}}$ is informative. However, this is not characteristic to inquisitive semantics in general: there are systems in which the "non-informativeness" requirement is abandoned. ${ }^{11}$

\subsection{Questions in IEL}

IEL adopts the "enrich with" approach to questions, although in its general setting does not predetermine how to shape them. On the level of a logical analysis it is assumed that questions are specific expressions of a formal language, and that to each question considered there is assigned an at least two-element set of direct answers. Direct answers are supposed to be declarative formulas (sentences in the first or higherorder case). In the general considerations it is also assumed that each finite and at least two-element set of declarative formulas/sentences constitutes the set of direct answers to a question of a formal language under consideration. One can build formal languages having the above features according to different patterns, for example by using Belnap's (Belnap and Steel 1976) or Kubiński's (Kubiński 1980) accounts of questions/interrogatives, or according to the semi-reductionistic schema proposed in Wiśniewski (1995) or Wiśniewski (2013).

In this paper we apply a formal account of propositional questions which is presumably the simplest one possible. ${ }^{12}$

\subsubsection{Questions as erotetic wffs}

We augment the vocabulary of $\mathcal{L}_{\mathcal{P}}$ with the following signs: ?, $\{$,$\} , and the comma.$ We get a new language, $\mathcal{L}_{\mathcal{P}}^{\text {? }}$, which has two categories of well-formed formulas: declarative well-formed formulas (d-wffs) and erotetic well-formed formulas, that is, questions.

A $d$-wff of $\mathcal{L}_{\mathcal{P}}^{?}$ is a wff of $\mathcal{L}_{\mathcal{P}}$.

A question of $\mathcal{L}_{\mathcal{P}}^{?}$ is an expression of the form:

$$
?\left\{A_{1}, \ldots, A_{n}\right\}
$$

where $n>1$ and $A_{1}, \ldots, A_{n}$ are nonequiform, that is, pairwise syntactically distinct, d-wffs of $\mathcal{L}_{\mathcal{P}}^{\text {? }}$ (i.e. wffs of $\mathcal{L}_{\mathcal{P}}$ ). Each of the d-wffs $A_{1}, \ldots, A_{n}$ is a direct answer to question (11), and these are the only direct answers to the question. Note that it is not assumed that direct answers must be true.

Each question of $\mathcal{L}_{\mathcal{P}}^{?}$ has at least two direct answers. At the same time the set of direct answers to a question is always finite. Note that questions of $\mathcal{L}_{\mathcal{P}}^{\text {? }}$ are wellformed expressions of the language. One cannot identify a question with the set of direct answers to it. For instance, $?\{p, q\} \neq ?\{q, p\}$.

\footnotetext{
11 This holds for the so-called presuppositional inquisitive semantics; see Ciardelli et al. (2013b, Section 6).

12 We follow Wiśniewski (1995, Chapter 3); see also Wiśniewski (2013, Chapter 2).
} 
An expression of the form (11) can be read:

Is it the case that $A_{1}$, or $\ldots$, or is it the case that $A_{n}$ ?

Let us stress: one should not confuse (12) with the grammatical form of naturallanguage questions formalized by it. The underlying idea is: an agent (a questioner or an answerer) regards some sentences as "principal possible answers", that is, answers providing neither less nor more information than the question under consideration calls for ("just-sufficient answers" for short), and these sentences are formally represented by the direct answers to the corresponding question of a formal language. $A_{1}, \ldots, A_{n}$ are simply the wffs which formalize just-sufficient answers differentiated in this way. ${ }^{13}$ However, in many cases what is regarded by an agent as "just-sufficient" depends not only on the form of the question, but, in addition, on contextual/pragmatic factors. It also happens that an agent does not see or bother that some of the answers regarded by him/her as just-sufficient entail other(s) (as in, e.g., "Who is John: a philosopher, or a philosopher or a theologian?") or even are logically equivalent. IEL differentiates such "faulty" questions at the semantic level, remaining very liberal at the level of syntax. For the sake of generality no objective, logical concept of "just-sufficiency" underlies the account of questions proposed: faulty questions asked by irrational agents are not ignored.

The schema (11) is general enough to capture most (if not all) of propositional questions studied in the literature.

Notation. We will use $Q, Q^{*}, Q_{1}, \ldots$ as metalanguage variables for questions of $\mathcal{L}_{\mathcal{P}}^{\text {? }}$. The set of direct answers to a question $Q$ will be referred to as $\mathrm{d} Q$.

For the sake of concision we adopt some notational conventions pertaining to questions of $\mathcal{L}_{\mathcal{P}}^{?}$ which we will frequently refer to. Questions of the form:

13 For example, any of the following:

$$
\begin{aligned}
& ?\{p, q\} \\
& ?\{p \wedge \neg q, \neg p \wedge q\} \\
& ?\{p \wedge \neg q, \neg p \wedge q, p \wedge q\} \\
& ?\{p, q, \neg(p \vee q)\}
\end{aligned}
$$

corresponds to a question expressed by the interrogative sentence:

Is John a philosopher, or a logician?

If (17) is understood so that a single choice among the alternatives given is sufficient for answering the relevant question, the question is represented by (13). If an exclusive choice is requested, the representation is (14). If a complete choice is requested, the question is represented by (15). If, however, the question expressed by (17) is construed as allowing that neither of the alternatives holds, it has (16) as its representation. 


$$
?\{A \wedge B, A \wedge \neg B, \neg A \wedge B, \neg A \wedge \neg B\}
$$

will be abbreviated as:

$$
? \pm|A, B|
$$

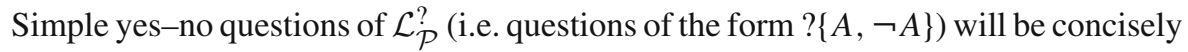
written as:

\section{3 "Translating" InqB-questions into questions of $\mathcal{L}_{\mathcal{P}}^{?}$}

Let $A$ be a d-wff having the $\mathcal{Q}$-property, that is, a question of $\mathcal{L}_{\mathcal{P}}$. One can always find a question $Q$ of $\mathcal{L}_{\mathcal{P}}^{?}$ such that the family of all the truth sets of direct answers to $Q$ is just the set of possibilities for $A$.

The idea is simple. Let $A$ be an inquisitive question of $\mathcal{L}_{\mathcal{P}}$. For simplicity, assume that $\mathcal{P}$ is a finite set. So the number of states is finite as well. $A$ is, by assumption, inquisitive, so the number of possibilities is greater than 1 , but still finite. We calculate the possibilities for $A$. Each possibility will be the truth set of some wff of $\mathcal{L}_{\mathcal{P}}{ }^{14}$ For each possibility obtained we choose exactly one wff such that the possibility is just the truth set of the wff. ${ }^{15}$ Since the possibilities are distinct, we get at least twoelement set of nonequiform d-wffs. Finally, we consider these d-wffs as direct answers to the corresponding question of $\mathcal{L}_{\mathcal{P}}^{\text {? }}$. For instance, (6) is the set of possibilities for (5). Therefore (5) translates, inter alia, into:

$$
?\{p, q, \neg(p \vee q)\}
$$

What about non-inquisitive questions of $\mathcal{L}_{\mathcal{P}}$ ? If $A$ is a non-inquisitive question of the language, $|A|$ equals $\mathcal{W}_{\mathcal{P}}$, since $A$ is a classical tautology. We choose a tautology, $B$, which is syntactically distinct from $A$. The question ? $\{A, B\}$ corresponds to $A .{ }^{16}$

\subsection{Proper questions of $\mathcal{L}_{\mathcal{P}}^{?}$ and inquisitiveness}

In this section we point out some close connections between the so-called proper questions and inquisitiveness. Proper questions constitute the most important semantic category of questions in IEL.

\footnotetext{
14 "Any formula can be represented as a disjunction of assertions in such a way that its possibilities coincide with the classical meaning of the disjuncts" (Ciardelli 2009, pp. 19-20.) The result is explicitly proven in Ciardelli (2009) for finite $\mathcal{P}$ 's, but, obviously, the finiteness assumption is dispensable. See also Lemma 6 in Sect. 4.3 .

15 Observe that $|\neg p| \cap|\neg q|$ is the truth set of both $\neg(p \vee q)$ and $\neg p \wedge \neg q$. In general, a possibility is always the truth set of many CL-equivalent wffs.

16 We owe this observation to an anonymous referee.
} 
We need some auxiliary notions.

Following Belnap (see Belnap and Steel 1976, pp. 119-120), we say that a d-wff $B$ is a presupposition of a question $Q$ iff $B$ is entailed by each direct answer to $Q$. By Pres $Q$ we designate the set of all the presuppositions of $Q$.

Definition 11 (Self-rhetorical question) A question $Q$ is self-rhetorical iff Pres $Q$ entails some direct answer(s) to $Q$.

The underlying idea is: a self-rhetorical question is a question that is already resolved by its presupposition(s).

As long as the language $\mathcal{L}_{\mathcal{P}}^{?}$ is concerned, 'entails' means 'CL-entails'. We have:

Corollary 3 Let $\mathrm{d} Q=\left\{A_{1}, \ldots, A_{n}\right\} . Q$ is self-rhetorical iff $\left|A_{1} \vee \cdots \vee A_{n}\right| \subseteq|A|$ for some $A \in \mathrm{d} Q$.

In the case of $\mathcal{L}_{\mathcal{P}}^{?}$ proper questions are defined by: ${ }^{17}$

Definition 12 (Proper question of $\mathcal{L}_{\mathcal{P}}^{?}$ ) A question $Q$ of $\mathcal{L}_{\mathcal{P}}^{?}$ is proper iff $Q$ is not self-rhetorical.

One can prove:

Lemma 2 If ? $\left\{A_{1}, \ldots, A_{n}\right\}$ is proper, then $A_{1} \vee \cdots \vee A_{n}$ is inquisitive.

Proof Assume that $A_{1} \vee \cdots \vee A_{n}$ is not inquisitive. Hence:

$$
\left|A_{1} \vee \cdots \vee A_{n}\right| \vDash A_{1} \vee \cdots \vee A_{n}
$$

Therefore for some $i$, where $1 \leq i \leq n$ :

$$
\left|A_{1} \vee \cdots \vee A_{n}\right| \vDash A_{i}
$$

But if $|B| \vDash A$, then $B \models_{c l} A$. Thus (23) yields:

$$
\left|A_{1} \vee \cdots \vee A_{n}\right| \subseteq\left|A_{i}\right|
$$

for some $1 \leq i \leq n$.

Note that Lemma 2 cannot be strengthened to equivalence. For consider the formula:

$$
(p \vee \neg p) \vee(q \vee \neg q)
$$

(25) is inquisitive, but ? $\{p \vee \neg p, q \vee \neg q\}$ is not a proper question.

\footnotetext{
17 Proper questions are defined in IEL as normal questions which are not self-rhetorical; a question is normal iff the set of its presuppositions is non-empty and mc-entails the set of direct answers to the question. Since each question of $\mathcal{L}_{\mathcal{P}}^{?}$ is normal, in the case of $\mathcal{L}_{\mathcal{P}}^{?}$ the definition simplifies.
} 
In order to go further we have to introduce the assertion operator, !. As in InqB, we define:

$$
! A={ }_{d f} \neg \neg A
$$

Note that $! A$ is always an assertion. Moreover, the following holds:

Corollary $4 \sigma \vDash !$ iff $\{w\} \vDash !$ f for each $w \in \sigma$.

We have: ${ }^{18}$

Lemma $3 ?\left\{A_{1}, \ldots, A_{n}\right\}$ is proper iff $! A_{1} \vee \cdots \vee ! A_{n}$ is inquisitive.

Proof For conciseness, let us abbreviate:

$$
! A_{1} \vee \cdots \vee ! A_{n}
$$

as $! A_{(1, n)}$.

$(\Rightarrow)$ Suppose that $! A_{(1, n)}$ is not inquisitive. Hence $\left|! A_{(1, n)}\right| \vDash ! A_{(1, n)}$. Thus $\left|! A_{(1, n)}\right| \vDash$ $! A_{i}$ for some $i$, where $1 \leq i \leq n$, and therefore $! A_{(1, n)} \models_{c l} ! A_{i}$. It follows that $A_{1} \vee \cdots \vee A_{n} \models_{c l} A_{i}$, that is, ? $\left\{A_{1}, \ldots, A_{n}\right\}$ is not proper.

$(\Leftarrow)$ If $! A_{(1, n)}$ is inquisitive, then $\left|! A_{(1, n)}\right| \not \mid ! A_{(1, n)}$. Hence $\left|! A_{(1, n)}\right| \not \forall ! A_{i}$ for $i=$ $1, \ldots, n$. Thus, by Corollary 4 , for each $i$, where $1 \leq i \leq n$, there exists $w \in\left|! A_{(1, n)}\right|$ such that $\{w\} \not \forall A_{i}$. Therefore $A_{1} \vee \cdots \vee A_{n} \not \models_{c l} A_{i}$ for $i=1, \ldots, n$, that is, ? $\left\{A_{1}, \ldots, A_{n}\right\}$ is proper.

Lemma 3 shows that a question of $\mathcal{L}_{\mathcal{P}}^{\text {? }}$ is proper if, and only if a disjunction of all its "asserted" direct answers is inquisitive. This sheds new light on the concept of proper question.

As for transitions from questions of $\mathcal{L}_{\mathcal{P}}^{\text {? }}$ to questions of $\mathcal{L}_{\mathcal{P}}$, Lemma 3 yields that a proper question of $\mathcal{L}_{\mathcal{P}}^{?}$ is akin to an inquisitive question of $\mathcal{L}_{\mathcal{P}}$ of the form (27) provided that the wff (27) is not informative (since each question of $\mathcal{L}_{\mathcal{P}}$ is, by definition, noninformative). ${ }^{19}$ Needless to say, in the case of some questions of $\mathcal{L}_{\mathcal{P}}^{\text {? }}$ the respective d-wffs falling under the schema (27) are informative (a simple example: ?\{p,q\}).

Terminology. From now on, unless otherwise stated, by questions we will mean questions of $\mathcal{L}_{\mathcal{P}}^{\text {? }}$, and similarly for d-wffs.

\section{Evocation of questions}

\subsection{Definition of evocation}

Evocation of questions is a binary relation between sets of d-wffs and questions. The idea underlying the definition of evocation proposed in IEL is very simple. A question

\footnotetext{
18 We owe this observation to a remark of an anonymous referee.

19 But recall that there are systems of inquisitive semantics in which this requirement is abandoned; see footnote 11 .
} 
$Q$ is evoked by a set of d-wffs $X$ when, and only when the truth of all the d-wffs in $X$ warrants the existence of a true direct answer to $Q$, but does not warrant the truth of any single direct answer to $Q$. In other words, if $X$ consists of truths, at least one direct answer to $Q$ must be true, but the issue "which one" remains unresolved.

The notion of multiple-conclusion entailment allows us to express the above intuition in exact terms. Let $\| \models$ stand for mc-entailment (see Sect. 2.3).

Definition 13 (Evocation) A set of d-wffs $X$ evokes a question $Q$ (in symbols: $\mathbf{E}(X, Q))$ iff

1. $X \|=\mathrm{d} Q$, and

2. $X \mid \not \models\{A\}$ for each $A \in \mathrm{d} Q$.

By putting $\| \models_{c l}$ in the place of $\| \models$ we get the definition of $C L$-evocation. It will be referred to as $\mathbf{E}_{c l}$.

In the case of $\mathcal{L}_{\mathcal{P}}^{?}$ (but not in the general case!) we can get rid of mc-entailment.

Corollary $5 \mathbf{E}_{c l}\left(X, ?\left\{A_{1}, \ldots A_{n}\right\}\right)$ iff $X \models_{c l} A_{1} \vee \cdots \vee A_{n}$ and $X \not \models_{c l}$ A for each $A \in\left\{A_{1}, \ldots A_{n}\right\}$.

Let us note:

Lemma 4 A question $Q$ of $\mathcal{L}_{\mathcal{P}}^{?}$ is proper iff $Q$ is $C L$-evoked by a certain set of $d$-wffs of $\mathcal{L}_{\mathcal{P}}^{?}$.

Proof Let $Q=?\left\{A_{1}, \ldots, A_{n}\right\}$.

Clearly, if $Q$ is proper, then $\mathbf{E}_{c l}\left(A_{1} \vee \cdots \vee A_{n}, Q\right)$.

If $\mathbf{E}_{c l}(X, Q)$, then $X \models_{c l} A_{1} \vee \cdots \vee A_{n}$ and $X \not \models_{c l} A$ for each $A \in \mathrm{d} Q$. Suppose that $Q$ is not proper. Therefore $A_{1} \vee \cdots \vee A_{n} \models_{c l} A$ for some $A \in \mathrm{d} Q$ and hence $X \models_{c l} A$. A contradiction.

\subsubsection{Examples of evocation}

As before, $p, q, r$ are propositional variables of $\mathcal{L}_{\mathcal{P}}$. For the sake of brevity, in what follows we use object-language expressions as their metalanguage names, and we characterize sets by listing their elements.

$$
\begin{aligned}
& \mathbf{E}_{c l}(p \vee \neg p, ? p) \\
& \mathbf{E}_{c l}(p \vee q, ? p) \\
& \mathbf{E}_{c l}(p \rightarrow q, ? p) \\
& \mathbf{E}_{c l}(p \rightarrow q, ? q) \\
& \mathbf{E}_{c l}(p \vee q, ?\{p, q\}) \\
& \mathbf{E}_{c l}(p \vee q, ?(p \wedge q)) \\
& \mathbf{E}_{c l}(p \rightarrow q \vee r, p, ?\{q, r\}) \\
& \mathbf{E}_{c l}(p \wedge q \rightarrow r, \neg r, ?\{\neg p, \neg q\}) \\
& \mathbf{E}_{c l}(p \wedge(q \vee r), ?\{p \wedge q, p \wedge r\})
\end{aligned}
$$


For properties of CL-evocation and further examples see, e.g., Wiśniewski (1995, 2001, 2013). For alternative accounts see Meheus (1999, 2001).

\subsection{Evocation and inquisitiveness}

One can easily prove that a disjunction of all the direct answers to an evoked question is always inquisitive. By Lemma 2 and Lemma 4 we immediately get:

Corollary 6 If ? $\left\{A_{1}, \ldots, A_{n}\right\}$ is $C L$-evoked by a certain set of $d$-wffs, then $A_{1} \vee \cdots \vee$ $A_{n}$ is inquisitive.

Corollary 6 cannot be strengthened to equivalence. But we have: ${ }^{20}$

Theorem 1 Let $?\left\{A_{1}, \ldots, A_{n}\right\}$ be a question of $\mathcal{L}_{\mathcal{P}}^{?}$. ?\{ $\left\{A_{1}, \ldots, A_{n}\right\}$ is $C L$-evoked by a certain set of $d$-wffs iff $! A_{1} \vee \cdots \vee ! A_{n}$ is inquisitive.

Proof By Lemmas 3 and 4.

\subsection{Inquisitiveness and evocation}

As for propositional InqB, each inquisitive wff "offers" at least two possibilities, that is, maximal states that support the wff. Moreover, the following hold:

Lemma 5 Let $A$ be a $d$-wff. If $w \in|A|$, then there is a possibility for $A$ such that $w$ belongs to this possibility.

As we have already observed (see footnote 7), Lemma 5 follows from some results established in Ciardelli (2009) and Ciardelli and Roelofsen (2011).

Lemma 6 If $A$ is an inquisitive $d$-wff, then: (a) the set of possibilities for $A$ is finite, and (b) for each possibility $\gamma$ for $A$ there exists a $d$-wff $B$ such that $|B|$ equals $\gamma$.

The above lemma follows from the existence of disjunctive normal forms in InqB. For details see Ciardelli (2009, p. 39), or Ciardelli and Roelofsen (2011, p. 69).

Let us first prove that a d-wff is inquisitive if, and only if it CL-evokes a yes-no question whose affirmative answer expresses a possibility for the d-wff.

Theorem 2 Let $|B|$ constitute a possibility for a $d$-wff $A$. $A$ is inquisitive iff $\mathbf{E}_{c l}(A, ? B)$.

Proof $(\Rightarrow)$ Clearly $A \| \models_{c l}\{B, \neg B\}$.

Since $|B|$ is a possibility for $A$, we have $|B| \vDash A$. If $|A| \subseteq|B|$, then, by Lemma 1 , $|A| \vDash A$. It follows that $A$ is not inquisitive. A contradiction. Therefore $A \not \models_{c l} B$ and hence $A \mid \not{ }_{c l}\{B\}$.

Now observe that when $A$ is inquisitive, each possibility for $A$ must be non-empty (because if $\emptyset$ is a possibility for $A$, it is the only possibility for $A$ ).

\footnotetext{
20 Similarly as in the case of Lemma 3, we owe this observation to an anonymous referee.
} 
Since $|B|$ is a possibility for $A$, we have $|B| \vDash A$. Suppose that $A \models_{c l} \neg B$. Thus $B \models{ }_{c l} \neg A$. Since $|B| \neq \emptyset$, it follows that there exists $w \in \mathcal{W}_{\mathcal{P}}$ such that $A$ is true in $w$ and its classical negation, $\neg A$, is true in $w$, which is impossible. Hence $A \not \nvdash_{c l} \neg B$ and thus $A \mid \not \models_{c l}\{\neg B\}$.

$(\Leftarrow)$ If $\mathbf{E}_{c l}(A, ? B)$, then $A \not \nvdash_{c l} B$ and $A \not \nvdash_{c l} \neg B$. Hence $|A| \neq \emptyset$ as well as $|B| \neq \emptyset$. Thus, by Lemma 5, there exist non-empty possibilities for $A$ and for $B$.

Assume that $|B|$ is the only possibility for $A$. Hence info(A) $=|B|$ and thus $|A|=|B|$. It follows that $A \models_{c l} B$ and therefore $A$ does not CL-evoke ?B. A contradiction. Thus $|B|$ is not the only possibility for $A$. It follows that $A$ is inquisitive.

Comment. Observe that $|B|$ in the assumption of Theorem 2 is an arbitrary possibility for $A$. Thus a philosophical comment on Theorem 2 is this: a d-wff is inquisitive if, and only if it raises (in the sense of CL-evocation) yes-no questions about possibilities for the d-wff. This sheds new light on the concept of inquisitiveness (in InqB).

Next, it can be proven that an inquisitive d-wff raises (i.e. CL-evokes) a question whose direct answers express all the possibilities for the d-wff.

Theorem 3 Let $A$ be an inquisitive $d$-wff. If $\left\{\left|B_{1}\right|, \ldots,\left|B_{n}\right|\right\}$ is the set of possibilities for $A$, then $\mathbf{E}_{c l}\left(A, ?\left\{B_{1}, \ldots, B_{n}\right\}\right)$.

Proof If $A$ is inquisitive, then the set of possibilities has at least two elements and, by Lemma 6, is a finite set. Moreover, again by Lemma 6, each possibility for $A$ is the truth set of some d-wff.

Suppose that $\left\{\left|B_{1}\right|, \ldots,\left|B_{n}\right|\right\}$ is the set of possibilities for $A$.

Let $w \in|A|$. Hence $\{w\} \vDash A$. By Lemma $5, w$ belongs to some possibility for $A$. Since $\left|B_{1}\right|, \ldots,\left|B_{n}\right|$ exhaust the set of possibilities for $A, w$ belongs to some $\left|B_{i}\right|$ and hence to $\left|B_{1} \vee \cdots \vee B_{n}\right|$. Therefore $A \models_{c l} B_{1} \vee \cdots \vee B_{n}$ and thus $A \mid \models{ }_{c l}\left\{B_{1}, \ldots, B_{n}\right\}$.

If $A$ is inquisitive and $\left|B_{i}\right|$, for $1 \leq i \leq n$, is a possibility for $A$, then $|A| \nsubseteq\left|B_{i}\right|$, and hence $A \mid \not \vee_{c l}\left\{B_{i}\right\}$.

Thus $\mathbf{E}_{c l}\left(A, ?\left\{B_{1}, \ldots, B_{n}\right\}\right)$ holds.

Comment. One can expect an inquisitive wff to raise a question "about" all the possibilities for the wff, that is, a question whose direct answers express all the possibilities for the formula. The claim of Theorem 3 amounts exactly to this, but the concepts used belong to IEL.

Let us now prove:

Theorem 4 Let $A$ be an inquisitive $d$-wff, and let $\left|B_{1}\right|,\left|B_{2}\right|$ constitute distinct possibilities for $A$. Then $\mathbf{E}_{c l}\left(A, ? \pm\left|B_{1}, B_{2}\right|\right)$.

Proof Since $A$ is inquisitive and $\left|B_{1}\right|,\left|B_{2}\right|$ are possibilities for $A$, it follows that $|A| \nsubseteq\left|B_{i}\right|$ for $i=1,2$. Hence $A \mid \not \ell_{c l}\left\{B_{i}\right\}$.

For conciseness, let us designate '? $\pm\left|B_{1}, B_{2}\right|$ ' by $Q^{*}$. Recall that $\mathrm{d} Q^{*}=\left\{B_{1} \wedge\right.$ $\left.B_{2}, B_{1} \wedge \neg B_{2}, \neg B_{1} \wedge B_{2}, \neg B_{1} \wedge \neg B_{2}\right\}$. It is easily seen that a disjunction of all the elements of $\mathrm{d} Q^{*}$ is a classical tautology. Therefore $A \| \models_{c l} \mathrm{~d} Q^{*}$.

Since $A \mid \nvdash_{c l}\left\{B_{i}\right\}$ for $i=1,2$, we have $A \mid \not \nvdash_{c l} C$ for any $C \in \mathrm{d} Q^{*}$ different from $\neg B_{1} \wedge \neg B_{2}$. Suppose that the latter is CL-entailed by $A$. It follows that $A \models_{c l} \neg B_{i}$ 
for $i=1,2$, and hence $B_{i} \models{ }_{c l} \neg A$. On the other hand, $\left|B_{i}\right| \vDash A$ and thus $B_{i} \models_{c l}$ $A$. Therefore $\left|B_{1}\right|=\emptyset$ and $\left|B_{2}\right|=\emptyset$. Hence $\left|B_{1}\right|=\left|B_{2}\right|$, which contradicts the assumption. Therefore $A \mid \forall \nvdash_{c l}\left\{\neg B_{1} \wedge \neg B_{2}\right\}$. Thus for each $C \in \mathrm{d} Q^{*}: A \mid \not \models_{c l}\{C\}$.

Generally speaking, ? $\pm\left|B_{1}, B_{2}\right|$ is a "partition" question that asks which of the possibilities offered by $A$ and expressed by $B_{1}, B_{2}$ holds, and allows both of them as well as neither of them to hold.

Comment. Direct answers to the CL-evoked questions referred to in the above theorems express either possibilities or boolean combinations of possibilities for the evoking (inquisitive) d-wffs. This is not a general rule, however. The following:

$$
\mathbf{E}_{c l}(p, ? q)
$$

provides a simple (counter)example. But questions about possibilities and/or their boolean combinations seem to constitute the most natural category of questions CLevoked/raised by inquisitive d-wffs.

\section{Erotetic implication}

Validity of erotetic inferences which have questions as premises and conclusions is defined in IEL by means of erotetic implication, being a ternary semantic relation between a question, a (possibly empty) set of d-wffs, and a question. By defining erotetic implication we explicate the intuitive notion "a question arises from a question on the basis of a set of declarative sentences".

\subsection{Intuitions}

Let us start with natural-language examples.

Example 1 The question:

What airline did Andrew travel by : BA, Ryanair, or Air France?

along with:

Andrew travelled by BA or Air France if, and only if he arrived in

the morning, and by Ryanair if, and only if he arrived in the evening.

(erotetically) imply the question:

When did Andrew arrive : in the morning, or in the evening?

Example 2 The question:

Is Andrew lying? 
together with:

Andrew lies if, and only if he speaks very slowly.

imply:

Does Andrew speak very slowly?

Example 3 The question:

Did Andrew travel by BA, or by Ryanair, or by neither?

implies:

Did Andrew travel by BA?

What do the above examples have in common? First, the following holds for all of them:

(F) If at least one direct answer to an implying question is true and the set of declarative premises consists of truths, it is impossible that every direct answer to the implied question is false.

A question is sound iff at least one direct answer to it is true, and unsound otherwise. Thus $\left(\mathbf{F}_{1}\right)$ can be concisely expressed as:

$\left(\mathbf{F}_{1}{ }^{*}\right)$ If the implying question is sound and the declarative premises are true, it is impossible that the implied question is unsound.

As for Examples 2 and 3, condition $\left(\mathbf{F}_{1}\right)$ is satisfied for trivial reasons, since the implied questions are safe yes-no questions. But in the case of Example 1 it is fulfilled non-trivially: although Andrew might have arrived neither in the morning nor in the evening, this becomes impossible assuming that at least one direct answer to question (38) is true and the premise (39) is true.

The set of direct answers to a question can be thought of as determining the prospective "search space" (or "class of relevant options", if you prefer) required to be narrowed down, in the optimal case to a singleton set. The second property shared by the examples is the following:

$\left(\mathbf{F}_{2}\right)$ Any expansion of the set of declarative premises by a direct answer to the implied question results in narrowing down the initial search space determined by (the set of direct answers to) the implying question.

For instance, if we expand the (set of) declarative premises of Example 1 by the answer "In the morning" to the (implied) question (40), the remaining class of options comprises BA and Air France, while the answer "In the evening" leaves a singleton option, namely Ryanair. The case of Example 2 is even simpler, because the affirmative answer to the implied question entails, along with the declarative premise, the affirmative answer to the implying question, and similarly for negative answers. As 
for Example 3, the affirmative answer to the implied question resolves the implying question, while after receiving the negative answer the initial three-element class of options becomes narrowed down to a two-element class.

IEL defines erotetic implication in such a way that the effects sketched above occur due to purely semantic reasons.

\subsection{Definition of erotetic implication}

As in Sect. 4, $\|=$ stands for mc-entailment. Let us abbreviate ' $X \cup\{C\}$ ' as ' $X, C$ '. Recall that $\mathrm{d} Q$ designates the set of direct answers to $Q$. Here is the schema of definition of erotetic implication:

Definition 14 (Erotetic implication) A question $Q$ implies a question $Q_{1}$ on the basis of a set of d-wffs $X$ (in symbols: $\operatorname{Im}\left(Q, X, Q_{1}\right)$ ) iff:

1. for each $A \in \mathrm{d} Q: X, A \| \mathrm{d} Q_{1}$, and

2. for each $B \in \mathrm{d} Q_{1}$ there exists a non-empty proper subset $Y$ of $\mathrm{d} Q$ such that $X, B \|=Y$.

Note that the respective $Y$ 's can (but need not) be singleton sets. Moreover, we do not assume that $X \neq \emptyset$.

By putting $\| \models_{c l}$ for $\| \models$ we get the definition of classical erotetic implication, $\mathbf{I m}_{c l}$. Notation. For $Z=\left\{C_{1}, \ldots, C_{n}\right\}$, ' $\bigvee Z$ ' abbreviates ' $C_{1} \vee \cdots \vee C_{n}$ '. Of course, $\bigvee\left\{C_{1}\right\}=C_{1}$.

As for $\mathcal{L}_{\mathcal{P}}^{?}$ (but, again, not in the general case!), one can define erotetic implication without making use of the concept of mc-entailment. This is due to:

Corollary $7 \mathbf{I m}_{c l}\left(Q, X, Q_{1}\right)$ iff

1. $X, \bigvee \mathrm{d} Q \models{ }_{c l} \bigvee \mathrm{d} Q_{1}$, and

2. for each $B \in \mathrm{d} Q_{1}$ there exists a non-empty proper subset $Y$ of $\mathrm{d} Q$ such that $X, B \models{ }_{c l} \bigvee Y$.

It is easily seen that clause (1) of Definition 14 warrants the fulfilment of condition $\left(\mathbf{F}_{1}\right)$. Concerning condition $\left(\mathbf{F}_{2}\right)$ : due to clause (2) of Definition 14, the set of declarative premises extended by any direct answer to the implied question mc-entails a proper subset of the set of direct answers to the implying question (or, to put it differently, entails either a direct answer or a disjunction of only some direct answers to the question). So if a given direct answer to the implied question is true and $X$ consists of truths, the initial class of options offered by the implying question becomes narrowed down, that is, roughly, there is a warranty that a true answer to the implying question belongs to a certain class that is "smaller" than the initial class. ${ }^{21}$ What is important, this condition holds for any direct answer to the implied question. Needless to say, the respective proper subsets related to different answers are usually distinct.

21 Let us stress that narrowing down is not tantamount to elimination. For example, let $Z=\{p, p \rightarrow q \vee r\}$ and $W=\{q, r, t\}$. Clearly, $Z \|=_{c l}\{q, r\}$ and $\{q, r\}$ is a proper subset of $W$, but $Z$ does not eliminate any d-wff in $W$. Similarly, elimination need not yield narrowing down. For instance, $\neg q$ eliminates the d-wff $q$ of $W$, but does not mc-entail (in CL) any proper subset of $W$. 


\subsubsection{Examples of erotetic implication}

In presenting examples of erotetic implication we adopt similar conventions as in Sect. 4.1.1. However, we use metalanguage variables for d-wffs and metalanguage schemata instead of d-wffs themselves.

$$
\begin{aligned}
& \operatorname{Im}_{c l}(? \neg A, ? A) \\
& \operatorname{Im}_{c l}(? A, ? \neg A) \\
& \operatorname{Im}_{c l}(? \pm|A, B|, ? A) \\
& \operatorname{Im}_{c l}(? \pm|A, B|, ? B) \\
& \operatorname{Im}_{c l}(? A, A \rightarrow B, B \rightarrow A, ? B) \\
& \operatorname{Im}_{c l}(? A, B \rightarrow A, C \rightarrow \neg A, B \vee C, ?\{B, C\}) \\
& \operatorname{Im}_{c l}\left(?\left\{A_{1}, \ldots, A_{n}\right\}, A_{1} \vee \cdots \vee A_{n}, ? A_{j}\right)
\end{aligned}
$$

where $1 \leq j \leq n$.

Notation. For $\left\{A_{1}, \ldots, A_{n}\right\}, ' \bigvee A_{(j, k)}$ ' abbreviates ' $A_{j} \vee A_{j+1} \vee \cdots \vee A_{k}$ '.

$$
\operatorname{Im}_{c l}\left(?\left\{A_{1}, \ldots, A_{n}\right\}, B \rightarrow \bigvee A_{(1, i-1)}, \neg B \rightarrow \bigvee A_{(i, n)}, ? B\right)
$$

where $1<i \leq n$.

$$
\mathbf{I m}_{c l}\left(?\left\{A_{1}, \ldots, A_{n}\right\}, B \rightarrow \bigvee A_{(1, i-1)}, C \rightarrow \bigvee A_{(i, n)}, B \vee C, ?\{B, C\}\right)
$$

where $1<i \leq n$.

For further examples, properties, and types of erotetic implication see Wiśniewski (1994, 1995, 2001, 2013). Let us add that erotetic implication links questions in the so-called erotetic search scenarios ${ }^{22}$ and constitutes the background of the method of Socratic proofs. ${ }^{23}$

\subsection{Erotetic implication and possibilities}

It can be proven that each question erotetically implies a question whose direct answers express all the possibilities for the direct answers to the implying question.

Notation. Let $\operatorname{Poss}(A)$ stand for the set of possibilities for a d-wff $A$.

Recall that when $A$ is inquisitive, then, by Lemma $6, \operatorname{Poss}(A)$ is a finite at least two-element set which comprises truth sets of some d-wffs. If $A$ is not inquisitive, then $\operatorname{Poss}(A)=\{|A|\}$.

Let us observe that each truth set may be expressed by more than one d-wff (in fact, by infinitely many d-wffs). For if $A$ and $B$ are CL-equivalent, then $|A|=|B|$, even if $A$ and $B$ are syntactically distinct. Thus we may assume without loss of generality that

\footnotetext{
22 For this concept see, e.g., Wiśniewski (2003), and Wiśniewski (2013, Part III).

23 For most recent developments of the method see Leszczyńska-Jasion et al. (2013).
} 
d-wffs $B_{1_{1}}, \ldots, B_{1_{k_{1}}}, \ldots, B_{n_{1}}, \ldots, B_{n_{k_{n}}}$ mentioned in the statement of Theorem 5 below are syntactically distinct.

Theorem 5 Let $\mathrm{d} Q=\left\{A_{1}, \ldots, A_{n}\right\}$ and let $\operatorname{Poss}\left(A_{i}\right)=\left\{\left|B_{i_{1}}\right|, \ldots,\left|B_{i_{k_{i}}}\right|\right\}$. Then $\operatorname{Im}_{c l}\left(Q, Q^{\diamond}\right)$, where $\mathrm{d} Q^{\diamond}=\left\{B_{1_{1}}, \ldots, B_{1_{k_{1}}}, \ldots, B_{n_{1}}, \ldots B_{n_{k_{n}}}\right\}$.

Proof If $A_{i}(1 \leq i \leq n)$ is inquisitive, then for reasons indicated in the proof of Theorem 3 we have $\bar{A}_{i} \|=_{c l}\left\{B_{i_{1}}, \ldots, B_{i_{k_{i}}}\right\}$ and hence $A_{i} \| \models_{c l} \mathrm{~d} Q^{\diamond}$. If $A$ is not inquisitive, then $A_{i} \| \models_{c l}\left\{B_{i_{1}}\right\}$ and therefore, again, $A_{i} \| \models_{c l} \mathrm{~d} Q^{\diamond}$.

Since each $\left|B_{i_{j}}\right|$ is a possibility for $A_{i}$ (where $1 \leq i \leq n, 1 \leq j \leq k_{i}$ ), we have $\left|B_{i_{j}}\right| \vDash A_{i}$ and therefore $B_{i_{j}} \| \models_{c l}\left\{A_{i}\right\}$.

Thus a (propositional) question is, in a sense, reducible to a question whose direct answers display all the possibilities for the direct answers to the initial question. Recall that if $|B|$ is a possibility for $A$, then $B$ CL-entails $A$. Thus when the implied question is answered, the implying question is answered as well.

Needless to say, the "questions about all the possibilities" are not the only questions erotetically implied.

\section{Concluding remarks}

As we have shown, enriching the conceptual apparatus of IEL with the notion of inquisitiveness results in revealing some important features of CL-evoked questions: disjunctions of all their direct answers are inquisitive (cf. Corollary 6) and CL-evoked questions are just these questions whose disjunctions of asserted direct answers are inquisitive (Theorem 1). On the other hand, when we "liberalize" the concept of question present in InqB, inquisitiveness of a formula amounts to evoking by the formula a yes-no question with the affirmative answer expressing a possibility for the formula (Theorem 2), and inquisitive formulas CL-evoke, among others, questions about all the possibilities for the formulas (Theorem 3). Moreover, a question is, in a sense, reducible to a question whose direct answers express all the possibilities for the direct answers to the "initial" question (Theorem 5). What is more important than the above formal results, however, is the inferential perspective gained: we are able to model the phenomenon of arriving at inquisitive questions and/or questions about possibilities.

Open Access This article is distributed under the terms of the Creative Commons Attribution License which permits any use, distribution, and reproduction in any medium, provided the original author(s) and the source are credited.

\section{References}

Belnap, N. D., \& Steel, T. P. (1976). The logic of questions and answers. New Haven: Yale University Press. Carnap, R. (1950). Logical foundations of probability. Chicago, IL: The University of Chicago Press. Ciardelli, I. (2009). Inquisitive semantics and intermediate logics. Master thesis, University of Amsterdam. 
Ciardelli, I., Groenendijk, J., \& Roelofsen, F. (2013a). Towards a logic of information exchange. An inquisitive witness semantics. In N. Bezhanishvili, et al. (Eds.), Logic, language, and computation: Revised selected papers from the 9th international Tbilisi symposium on language, logic, and computation. Springer, pp. 51-72.

Ciardelli, I., Groenendijk, J., \& Roelofsen, F. (2013b). On the semantics and logic of declaratives and interrogatives. Synthese doi:10.1007/s11229-013-0352-7.

Ciardelli, I., \& Roelofsen, F. (2011). Inquisitive logic. Journal of Philosophical Logic, 40, 55-94.

Ciardelli, I., \& Roelofsen, F. (2014). Inquisitive dynamic epistemic logic. Synthese. doi:10.1007/ s11229-014-0404-7.

Ginzburg, J. (2011). Questions: Logic and interactions. In J. van Benthem \& A. ter Meulen (Eds.), Handbook of logic and language (2nd ed., pp. 1133-1146). Elsevier.

Groenendijk, J. (2011). Erotetic languages and the inquisitive hierarchy. In J. van der Does \& C. Dutilh Novaes (Eds.), This is not a Festschift_Festschrift for Martin Stokhof. https://sites.google.com/site/ inquisitivesemantics/papers-1/publications.

Groenendijk, J., \& Roelofsen, F. (2009). Inquisitive semantics and pragmatics. In J. M. Larrazabal \& L. Zubeldia (Eds.), Meaning, content and argument. Proceedings of the ILCLI international workshop on semantics, pragmatics and rhetoric (pp. 41-72). University of the Basque Country Publication Service.

Groenendijk, J., \& Stokhof, M. (1997). Questions. In J. van Benthem \& A. ter Meulen (Eds.), Handbook of logic and language. New York: North Holland (pp. 1055-1125).

Groenendijk, J., \& Stokhof, M. (2011). Questions. In J. van Benthem \& A. ter Meulen (Eds.), Handbook of Logic and Language (2nd ed., pp. 1059-1132). Amsterdam: Elsevier.

Harrah, D. (2002). The logic of questions. In D. Gabbay, \& F. Guenthner (Eds.), Handbook of philosophical logic (Vol. 8, 2nd ed., pp. 1-60). Dordrecht: Kluwer.

Krifka, M. (2011). Questions. In K. von Helsinger, C. Maieborn \& P. Portner (Eds.), Semantics. An international handbook of natural language meaning. (Vol. II, pp. 1742-1785). Berlin: Mouton de Gruyter.

Kubiński, T. (1980). An outline of the logical theory of questions. Berlin: Akademie-Verlag.

Leszczyńska-Jasion, D., Urbański, M., \& Wiśniewski, A. (2013). Socratic trees. Studia Logica, 101(5), 959-986.

Meheus, J. (1999). Erotetic arguments from inconsistent premises. Logique et Analyse, 165-167, 49-80.

Meheus, J. (2001). Adaptive logic for question evocation. Logique et Analyse, 173-175, 135-164.

Roelofsen, F. (2013). Algebraic foundations for the semantic treatment of inquisitive content. Synthese, 190(1), 79-102.

Shoesmith, D. J., \& Smiley, T. J. (1978). Multiple-conclusion logic. Cambridge, MA: Cambridge University Press.

Wiśniewski, A. (1994). Erotetic implications. Journal of Philosophical Logic, 23, 174-195.

Wiśniewski, A. (1995). The posing of questions: Logical foundations of erotetic inferences. Dordrecht: Kluwer.

Wiśniewski, A. (1996). The logic of questions as a theory of erotetic arguments. Synthese, 109, 1-25.

Wiśniewski, A. (2001). Questions and inferences. Logique et Analyse, 173-175, 5-43.

Wiśniewski, A. (2003). Erotetic search scenarios. Synthese, 134, 389-427.

Wiśniewski, A. (2013). Questions, inferences, and scenarios. London: College Publications.

Wiśniewski, A. (2015). Semantics of questions. In S. Lappin \& C. Fox (Eds.), The handbook of contemporary semantic theory (2nd ed.). Boston: Wiley-Blackwell. 